\title{
Bilateral versus ipsilesional cortico- subcortical activity patterns in stroke show hemispheric dependence
}

International Journal of Stroke 0(0) 1-11

(C) 2018 World Stroke Organization Reprints and permissions: sagepub.co.uk/journalsPermissions.nav DOI: $10.1177 / 1747493018767164$ journals.sagepub.com/home/wso (\$)SAGE

\author{
Ana C Vidal ${ }^{1,2}$, Paula Banca ${ }^{3}$, Augusto G Pascoal', \\ Gustavo Cordeiro ${ }^{4}$, João Sargento-Freitas ${ }^{4}$ (D), Ana Gouveia ${ }^{4}$ \\ and Miguel Castelo-Branco ${ }^{2}$
}

\begin{abstract}
Background: Understanding of interhemispheric interactions in stroke patients during motor control is an important clinical neuroscience quest that may provide important clues for neurorehabilitation. In stroke patients bilateral overactivation in both hemispheres has been interpreted as a poor prognostic indicator of functional recovery. In contrast, ipsilesional patterns have been linked with better motor outcomes.
\end{abstract}

Aim: We investigated the pathophysiology of hemispheric interactions during limb movement without and with contralateral restraint, to mimic the effects of constraint-induced movement therapy. We used neuroimaging to probe brain activity with such a movement-dependent interhemispheric modulation paradigm.

Methods: We used a functional magnetic resonance imaging block design during which the plegic/paretic upper limb was recruited/mobilized to perform unilateral arm elevation, as a function of presence versus absence of contralateral limb restriction ( $n=20$, with balanced left/right lesion sites).

Results: Analysis of 10 right hemispheric stroke participants yielded bilateral sensorimotor cortex activation in all movement phases in contrast with the unilateral dominance seen in the 10 left hemispheric stroke participants. Superimposition of contralateral restriction led to a prominent shift from activation to deactivation response patterns, in particular in cortical and basal ganglia motor areas in right hemispheric stroke. Left hemispheric stroke was, in general, characterized by reduced activation patterns, even in the absence of restriction, which induced additional cortical silencing.

Conclusion: The observed hemispheric-dependent activation/deactivation shifts is novel and these pathophysiological observations suggest short-term neuroplasticity that may be useful for hemisphere-tailored neurorehabilitation.

\section{Keywords}

Stroke, interhemispheric interactions, physiotherapy, motor control, handedness, functional magnetic resonance imaging

Received: I October 2015; accepted: 6 July 2016

\section{Introduction}

Neurorehabilitation of motor deficits of the upper limb after a stroke episode often shows poor results. ${ }^{1,2}$ Although stroke is an important cause of mortality ${ }^{3}$ its survival rate is increasing leading to important challenges in the field of rehabilitation. ${ }^{4-8}$

The problem needs to be analyzed from its onset, in the acute phase of the stroke, in particular in which concerns neurophysiological adaptations and the window for plasticity. It is indeed important to understand the rules that determine changes in modulation properties of neural circuits and in neuroplasticity. ${ }^{9,10}$
In stroke patients the cortical activation of the ipsilesional hemisphere has been suggested to be associated with better outcomes in recovery of motor functions. ${ }^{11}$

\footnotetext{
'Fac Motricidade Humana, Universidade de Lisboa, CIPER, LBMF, Lisbon, Portugal

${ }^{2}$ Garcia de Orta Hospital, Almada, Portugal

${ }^{3}$ Faculty of Medicine, Visual Neuroscience Laboratory, CIBIT, IBILI, University of Coimbra, Coimbra, Portugal

${ }^{4}$ Department of Neurology, Stroke Unit, Coimbra University Hospital, Coimbra, Portugal

Corresponding author:

Miguel Castelo-Branco, Faculty of Medicine, Visual Neuroscience Laboratory, IBILI, University of Coimbra, Coimbra, Portugal.

Email: mcbranco@fmed.uc.pt
} 
In contrast, overactivation or increase of the contralesional hemisphere has been associated with sustained neurologic and motor deficits. ${ }^{12,13}$

Motor compensations maybe expressed by hyperactivation of the contralesional hemicorpus, as motor recruitment of the trunk has been linked to less available recruitment of the more affected upper limb, limiting recovery. ${ }^{14}$ In contrast, motor selective/specific patterns of compensation ${ }^{15}$ were reported to be associated with better outcomes for rehabilitation. ${ }^{16}$ Specific approaches, such as constraint-induced movement therapy (CIMT), use motor restriction of the contralateral upper limb (currently applied only to the hand) to help the paretic arm to redevelop lost motor functions. In longitudinal studies, the CIMT was reported to be able to change neural circuitry patterns from bilateral cortex motor activation to ipsilesional cortical activation. ${ }^{17,18}$ In any case, the use of the dichotomy of motor restriction versus facilitation has traditionally been used in physiotherapy, although its neuroscientific basis needs additional support. ${ }^{19,20}$

It is important to understand the physiological effect that results from the isolated restriction of the superior limb, as implemented by CIMT. If such restriction is maintained in time it becomes a "constraint technique." Here, by studying the physiological effects of such restriction, we aimed to provide a biological basis for such technique and its effective promotion of recovery of the "bad limb" in a hemispheric-dependent manner.

We posit that it is very important to study movement-dependent brain activation/deactivation patterns to better understand mechanisms underlying motor control. ${ }^{21-25}$ Different action goals and movement modulation (inhibition or facilitation) provide potentially relevant aspects to consider in rehabilitation. ${ }^{26}$ Here, we investigated the role of interhemispheric interactions in motor control and brain activity regulation as a function of inhibition/restriction of upper limb motion, as applied to stroke patients. By testing procedures usually applied in the physiotherapy in stroke patients, one might gain insights into the effects with potential influence on optimization of motor recovery. Here, neural modifications were studied on a shortterm basis after the restriction manipulation as a prior step for studies of long-term effects of procedures often applied in physiotherapy sessions.

\section{Methods}

\section{Participants}

Twenty stroke patients (12 female/eight male; age: 68.3 mean \pm 10.04 years), all right handed according to the Edinburg Handedness scale, ${ }^{27}$ participated in this single arm, within-subject study design. Data concerning patterns observed in normal control participants are described in a previous report. ${ }^{26}$ All patients had only one first clinical episodic of stroke prior to the study (see Table 1, which summarizes clinical and demographic data). This project was approved by the ethics committee of Faculty of Medicine, University of Coimbra, Portugal. All participants or family gave written informed consent, prior to their participation, according to the Declaration of Helsinki.

Patients were selected by team members of the Stroke Unit, Department of Neurology, Coimbra University Hospital. Inclusion criteria included the ability to understand and execute the motor task evaluated in this study. Stroke patients with clinical unstable (due to causes such as respiratory infection and fractures) or education level below full literacy were excluded.

The acute stroke group was evaluated after the first clinic episodic on average after $10.2 \pm 4.3$ days, with stroke location in territory cerebral middle artery. Left and right stroke patients were matched (all comparisons for the following variables not significant): in the right hemisphere stroke group, the following data were obtained: age $67 \pm 10.1$ years, volume area of territory cerebral middle artery: $45.8 \pm 54.6 \mathrm{~cm}^{3}$, National Institutes of Health Stroke Scale (NIHSS): 14.5 \pm 5.9 , and score of the Chedoke McMaster Stroke Assessment of the arm: $3.4 \pm 2.7$. In the left hemisphere stroke group data were as follows: age $69.4 \pm 8.8$ years, volume area of territory cerebral middle artery $46.8 \pm 53.3 \mathrm{~cm}^{3}$, NIHSS: $11.3 \pm 5.9$, and score of the Chedoke McMaster Stroke Assessment of the arm was $4.3 \pm 2.9$.

In both right and left hemisphere groups we had similar distributions in terms of stroke severity as quantified by NIHSS and motor shoulder incapacity as assessed by the Portuguese version of Chedoke McMaster stroke assessment scale. Concerning stroke affecting the right hemisphere, and according to the NIHSS we had the following distribution in terms of stroke severity: one minor, six moderate, and three moderate to severe. In terms of the evaluation of motor ability and impairment, and according to the Chedoke McMaster Stroke scale for the arm we had four hemiplegic cases and six hemiparetic conditions. Concerning stroke affecting the left hemisphere, and according to NIHSS we had the following distribution in terms of stroke severity: five moderate, three moderate to severe, and two severe. According to the Chedoke McMaster Stroke scale for the arm we had four hemiplegic cases and six hemiparetic conditions.

\section{Magnetic resonance imaging (MRI) scanning}

The stroke lesion was quantified by the $\mathrm{ABC} / 2$ formula $^{28}$ using the flair sequence of MRI (SyngoFast 
Table I. Clinical/demographic characteristics of stroke patients

\begin{tabular}{|c|c|c|c|c|c|c|c|c|c|}
\hline \multirow[b]{2}{*}{ Patient } & \multirow[b]{2}{*}{$\begin{array}{l}\text { Stroke } \\
\text { side }\end{array}$} & \multirow[b]{2}{*}{$\begin{array}{l}\text { Days of } \\
\text { stroke }\end{array}$} & \multirow{2}{*}{$\begin{array}{l}\text { Lesion } \\
\text { Volume } \\
\left(\mathrm{cm}^{3}\right)\end{array}$} & \multirow[b]{2}{*}{$\begin{array}{l}\text { Hemiplegic/ } \\
\text { hemiparetic side }\end{array}$} & \multirow[b]{2}{*}{ Age } & \multirow[b]{2}{*}{ Sex } & \multirow[b]{2}{*}{$\begin{array}{l}\text { NIHSS } \\
\text { initial }\end{array}$} & \multicolumn{2}{|c|}{$\begin{array}{l}\text { Chedoke } \\
\text { McMaster scale }\end{array}$} \\
\hline & & & & & & & & $\begin{array}{l}\text { Shoulder } \\
\text { pain }\end{array}$ & Arm \\
\hline No. I & Right & 8 & 76 & Left hemiplegic & 49 & $\mathrm{~F}$ & 19 & 6 & I \\
\hline No. 2 & Right & 23 & 25 & Left hemiparetic & 82 & $\mathrm{~F}$ & 6 & I & 7 \\
\hline No. 3 & Right & 7 & 21 & Left hemiparetic & 70 & $M$ & I & 7 & 5 \\
\hline No. 4 & Right & 9 & 91 & Left hemiparetic & 47 & $M$ & 19 & 7 & 7 \\
\hline No. 5 & Right & 9 & 75 & Left hemiplegic & 69 & $M$ & 12 & 7 & 7 \\
\hline No. 6 & Right & 19 & I & Left hemiparetic & 73 & $M$ & 7 & 7 & I \\
\hline No. 7 & Right & 7 & I & Left hemiparetic & 70 & $\mathrm{~F}$ & 8 & 7 & 7 \\
\hline No. 8 & Right & 7 & 3.3 & Left hemiplegic & 70 & $M$ & 16 & 6 & 6 \\
\hline No. 9 & Right & 12 & 2.3 & Left hemiplegic & 69 & $M$ & 14 & 4 & I \\
\hline No. 10 & Right & 9 & 172 & Left hemiparetic & 75 & $M$ & 11 & 6 & I \\
\hline No. II & Left & 7 & 1.7 & Right hemiplegic & 72 & $\mathrm{~F}$ & 10 & 6 & 2 \\
\hline No. 12 & Left & 9 & 156 & Right hemiplegic & 80 & $\mathrm{~F}$ & 23 & 7 & 2 \\
\hline No. 13 & Left & 9 & 15.2 & Right hemiplegic & 78 & $\mathrm{~F}$ & 19 & 6 & 6 \\
\hline No. 14 & Left & 9 & 21 & Right hemiparetic & 63 & $\mathrm{~F}$ & II & 6 & 1 \\
\hline No. 15 & Left & 12 & 7.6 & Right hemiparetic & 76 & $\mathrm{~F}$ & 7 & 6 & I \\
\hline No. 16 & Left & 9 & 69 & Right hemiplegic & 54 & $\mathrm{~F}$ & 22 & 7 & 7 \\
\hline No. 17 & Left & 7 & 22 & Right hemiparetic & 74 & $\mathrm{~F}$ & 18 & 7 & 6 \\
\hline No. 18 & Left & 7 & 3.7 & Right hemiparetic & 57 & $\mathrm{~F}$ & 7 & 6 & I \\
\hline No. 19 & Left & 10 & 34 & Right hemiplegic & 77 & $\mathrm{~F}$ & 16 & 5 & 1 \\
\hline No. 20 & Left & 15 & 128 & Right hemiparetic & 61 & $\mathrm{~F}$ & 12 & 7 & 7 \\
\hline
\end{tabular}

F: female; M: male

National Institutes of Health Stroke Scale (NIHSS): score 0: no stroke symptoms; I-4: minor stroke; 5-15: moderate stroke; 16-20: moderateto-severe stroke; 2 I-42: severe stroke.

Chedoke McMaster Stroke Scale (impairment inventory: shoulder pain): Stage I more pain than Stage 5. Stage 6 no shoulder pain, but at least one negative prognostic indicator is present; Stage 7 shoulder pain and prognostic indicators are absent/(impairment inventory: stage of arm): Stage 7 better than stage I.

View (Siemens)). ${ }^{29}$ The $\mathrm{ABC} / 2$ method has the advantage of being objective, highly consistent across centers in large-scale studies. It is widely used in stroke units worldwide as an easily accessible evaluation procedure, with several studies showing similar accuracy as compared to planimetric analysis (for instance, Sims et al. ${ }^{28}$ : " $\mathrm{ABC} / 2$ for rapid clinical estimate of infarct, perfusion, and mismatch volumes").

\section{Functional magnetic resonance imaging (fMRI)-Motor paradigm}

All participants underwent one structural magnetic resonance scan and two fMRI scanning sessions: (1) the dominant upper limb was restrained while the nondominant upper limb performed an arm elevation (AE); (2) the opposite stimulation pattern was applied 
(nondominant restrained upper limb and dominant facilitation of $\mathrm{AE}$ ).

\section{Sequence of motor paradigm}

The sequence of the motor paradigm was composed of five $30 \mathrm{~s}$ blocks. The first condition consisted of a simple facilitation of AE. The second condition was a combination of the facilitation of the AE plus the contralateral upper limb restraining ( $\mathrm{AE}+\mathrm{LR})$ inhibiting its motor action. All blocks were subdivided in three periods of $10 \mathrm{~s}$. In total, there were 15 periods, repeated 10 times (cycle repetitions) in a random order. A scheme of the fMRI experimental design is described in Figure 1.

\section{Detailed task description}

The task is described in detail elsewhere. ${ }^{26}$ The facilitation of AE refers to the arm flexion, at the glenohumeral joint, with the elbow in full extension. A customized Cellacast ${ }^{\circledR}$ splint was placed on the anterior part of arm and forearm in order to ensure elbow extension (Figure 1C and F).

Near bore manual assistance by the researcher/ physiotherapist was applied to all subjects to help initialize/orient arm motion.

The facilitation of AE was defined as a motor action composed by three periods with $10 \mathrm{~s}$ each: upward, hold, and downward. To facilitate the movement, a mobilization was performed in assisting-active mode, in which the researcher/physiotherapist induced the movement. For each period, subjects heard verbal instructions indicating the motor activities.

$\mathrm{AE}$ is integrated in activities of daily living and the flexion of arm reflects a component of motion in the shoulder complex. Previous to a stroke the elevation of arm is integrated in automatic movements groups, given the repeated experience in executing them. For this reason, we used a strategy based on intermittent facilitation, with short speed boosts in the same way that automatic walk is promoted. This enabled an overall similar pattern of stimulation/facilitation across subjects.

The rest periods had two types of position: (1) the upper limbs were in neutral position, resting along the body; (2) one upper limb was in neutral position and the contralateral limb was restrained, with shoulder adduction, crossing over the middle line of trunk (Figure 1).

The contralateral limb restraint (LR) was achieved by keeping shoulder adduction, crossing the arm in such a way that the hand was over the contralateral pelvis. Customized abdominal and hand slings with Velcro ${ }^{\circledR}$ strips were used to ensure an efficient limb restriction and quick release. Thus, this promoted inhibition of muscle activity in the upper limb.

We used three periods involving restraint manipulations: (1) the limb is placed in the restraint position (adduction of shoulder with crossing the middle line of trunk), but it returns the neutral position; (2) the limb is placed in restraint position and stays (in a position of shoulder adduction); (3) the limb is released from the restraint position (starts in the adduction shoulder and returns to the neutral position).

Data acquisition. Magnetic resonance data were collected on a 3 Tesla Siemens Tim Trio. High-resolution anatomical images were acquired for each participant using a T1-weightd MPRAGE sequence $1 \mathrm{~mm} \times 1 \mathrm{~mm} \times$ $1 \mathrm{~mm}$ voxel size, repetition time (TR): $2300 \mathrm{~ms}$, echo time (TE): $2.98 \mathrm{~ms}$, flip angle (FA): $9^{\circ}$, field of view (FOV): $256 \mathrm{~mm}$. The fMRI for each shoulder elevation

Figure I. Schematic of the experimental design in fMRI experiments. Limb manipulation during the experimental blocks (and control contralateral motion or restraint positioning during mid-period in baseline) is depicted by arrow symbols.

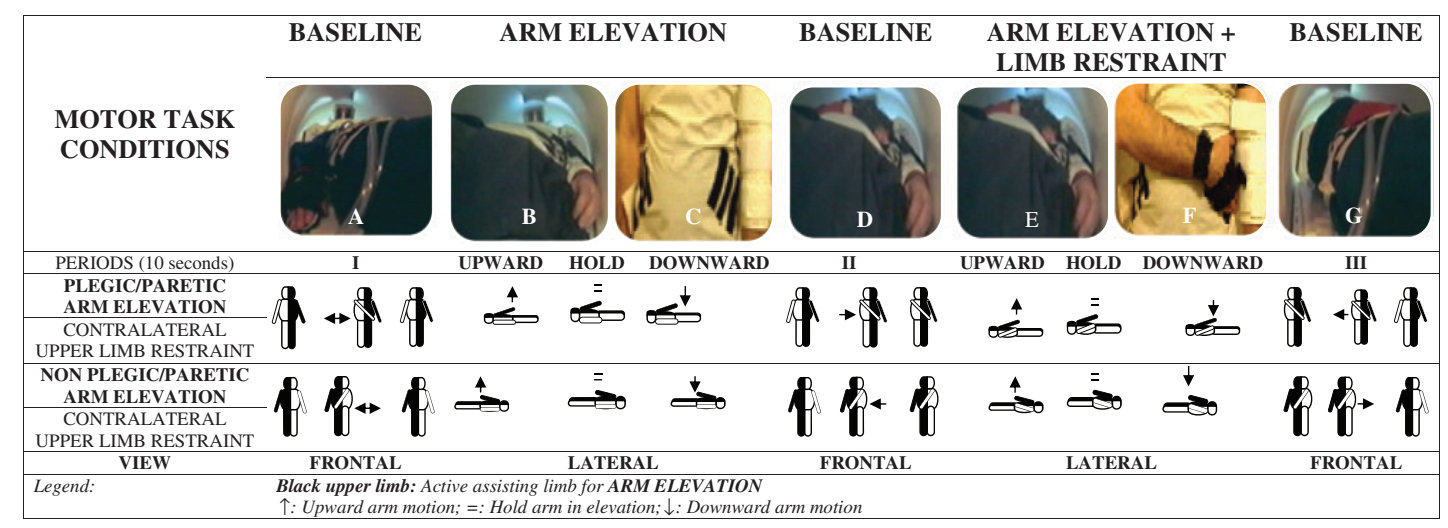


(dominant and nondominant) was obtained using a T2-weighted BOLD contrast echo planar imaging sequence $2.5 \mathrm{~mm} \times 2.5 \mathrm{~mm} \times 3 \mathrm{~mm}$ voxel size, TR: $3000 \mathrm{~ms}$, TE: $38 \mathrm{~ms}$, FOV: $256 \mathrm{~mm}$. During each experiment, T1-weighted anatomical images were collected first followed by the functional runs. Each set included 10 continuous scans for first run and second run.

\section{Image processing and data analysis}

The location of the stroke was previously defined to a region that received blood perfusion by middle cerebral artery vascular territory infarction. The volume of the ischemic area was measured using a brain structural magnetic resonance scan, in flair sequence, visualized by software SyngoFast View (Siemens), and quantified using the formula of $\mathrm{ABC} / 2$ (where $\mathrm{A}$ is the greatest hemorrhage diameter by $\mathrm{CT}, \mathrm{B}$ is the diameter $90^{\circ}$ to $\mathrm{A}$, and $\mathrm{C}$ is the approximate number of $\mathrm{CT}$ slices with hemorrhage multiplied by the slice thickness).

The imaging data analysis was performed using the Brain Voyager Software (QX version 2.4; Brain Innovation B.V., The Netherlands). Head motion was corrected and three-dimensional temporal filtering and slice scan time correction were performed. Maps were automatically registered into the standard Talairach space. Head motions $>2 \mathrm{~mm}$ implied subject exclusion. Movement of upper limbs was video monitored.

In the first-level analysis, data were analyzed for each subject separately using general linear models (GLMs) to identify significantly activated voxels. After model estimation, contrast images derived from each participant were calculated and analyzed individually. Then, a second-level analysis, using one-way repeated measures ANOVAs (within-group design), was conducted. In the first stage, wholevolume GLMs were computed and corrected for temporal serial correlations, for subsequent group inferences. Each fMRI session with tasks for dominant and nondominant shoulder elevation was then processed separately, using a random effects analysis. This allowed inferring whether the observed results might be generalized to the population. Statistical maps were corrected for multiple comparisons using the false discovery rate procedure for individual analysis in for BrainVoyager QX ${ }^{30}$ with $p<0.05$ and group analysis with $p>0.05$, with the Monte Carlo 1000 interactions. Cluster-size thresholding allowed for the definition of volumes of interest in relation to defined Brodmann regions.

Statistical models for region of interest (ROI) analysis. In order to compare the recruitment of brain regions induced by the contrast presence versus absence of contralateral LR during $\mathrm{AE}((\mathrm{AE}+\mathrm{LR})$ versus $(\mathrm{AE}))$ we first used the number of significant voxels in ROIs corresponding to sensorimotor cortex, basal ganglia, and cerebellum. For comparison we used, as stated above, the contrast analysis of (AE + LR) versus (AE), with $\mathrm{p}<0.05$ (see above).

\section{Results}

\section{Functional activation patterns}

We found different brain activity patterns between right and left hemisphere stroke during plegic/paretic AE. For nonplegic/paretic AEs we also found different patterns for the dominant and nondominant upper limbs. The addition of a contralateral upper limb restriction led to a deactivation in all conditions that was also hemisphere dependent.

Cortical bilateral activation only in right hemisphere stroke during plegic/paretic $A E$. In patients with right hemisphere stroke, cortical activation in bilateral sensorimotor cortex was found, during plegic/paretic AE (nondominant or left arm), especially in the supplementary motor area (Brodmann area 6) (Figure 2). Subcortically, we observed a contralesional activation pattern localized in left striatum, subthalamic, and red nucleus. The cerebellar activity was ipsilateral in the upward condition, during AE, and bilateral during the hold and downward periods (for more detailed information see Supplementary tables, Appendix 1, Supplementary Tables 1, 2, and 3).

Ipsilesional cortical activation only in left hemispheric stroke during upward plegic/paretic AE. In patients with left hemispheric stroke (Figure 3), we found a small ipsilesional cortical activation of sensorimotor cortex and ipsilateral cerebellum activation only during upward plegic/ paretic AE (dominant or right) (for more details see Supplementary Tables 4 and 5 in Supplementary tables, Appendix 1). During hold periods, we observed bilateral deactivations and during downward phases a bilateral activation.

Subcortically, we also found a deactivation of ipsilesional striatum, bilateral thalamus, and right red nucleus during upward and hold periods. During the downward period we observed dominance of bilateral activation in subcortical regions and cerebellum (Supplementary tables, Appendix 1 see Supplementary Tables 4 and 5).

Whole brain deactivation during $A E$ with contralateral limb restriction. In patients with right hemispheric stroke, the presence of dominant upper LR during plegic/ paretic AE results in the silence of contralesional cortical areas while maintaining ipsilesional activation of 
Figure 2. Statistical maps of group analysis of the right hemispheric stroke patients during plegic/paretic AE in presence/absence restriction of contralateral upper limb.

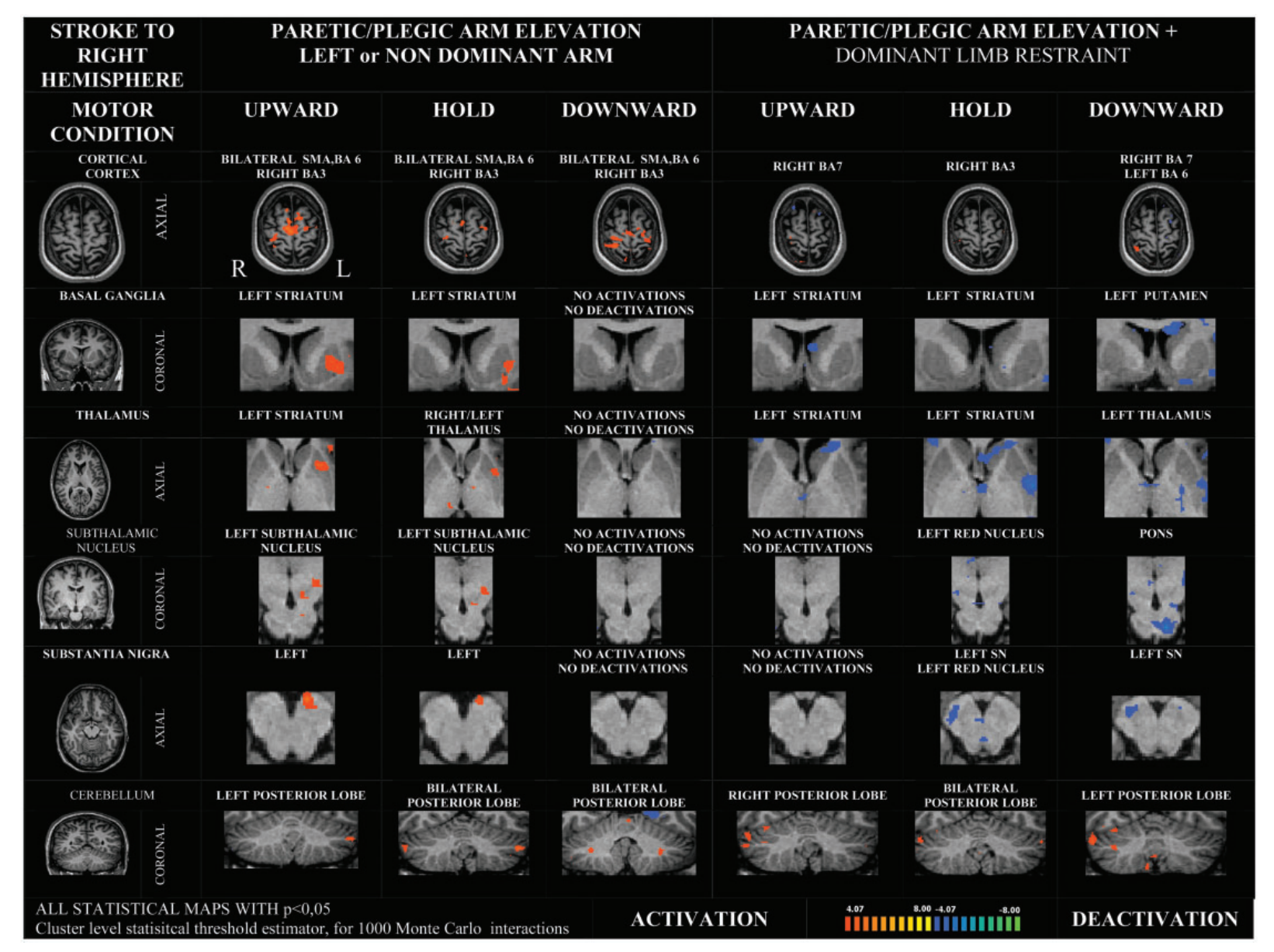

the sensorimotor cortex. The basal ganglia deactivated but cerebellar activity was similar in both conditions (with and without LR).

In patients with left hemispheric stroke, the nondominant upper LR during plegic/paretic AE resulted in bilateral reduction of cortical activation. During the hold phase, we found a deactivation of contralesional supplementary motor area and ipsilateral primary motor cortex. The subcortical pattern of activity was similar in both conditions of plegic/paretic AE. Concerning cerebellar activity changes activations predominated over deactivations.

In sum, concerning both nonplegic/paretic AEs (Figures 4 and 5) of dominant or nondominant upper limbs, when restraint of contralateral upper limb was added, we observed cortical, subcortical, and cerebellar deactivation. Only dominant AE was associated to contralateral cerebellar activation.

\section{Summary of results as a function of movement phase}

\section{Cortical deactivation is only present in dominant nonplegic/paretic $A E$}

Statistically significant deactivation of ipsilesional hemispheric or ipsilateral relative to nonplegic/paretic dominant AE was only observed during upward periods. During hold phases, this deactivation was stronger, extending to bilateral sensorimotor cortex.

Disparities between subcortical activity during nonplegic/paretic $A E$. During upward and hold periods, dominant AE was associated with deactivation of bilateral striatum and ipsilateral substantia nigra and red nucleus. The nondominant AE was associated with activation of contralateral striatum and bilateral substantia nigra. 
Figure 3. Statistical maps of group analysis of the left hemispheric stroke patients during plegic/paretic AE in presence/absence restriction of contralateral upper limb.

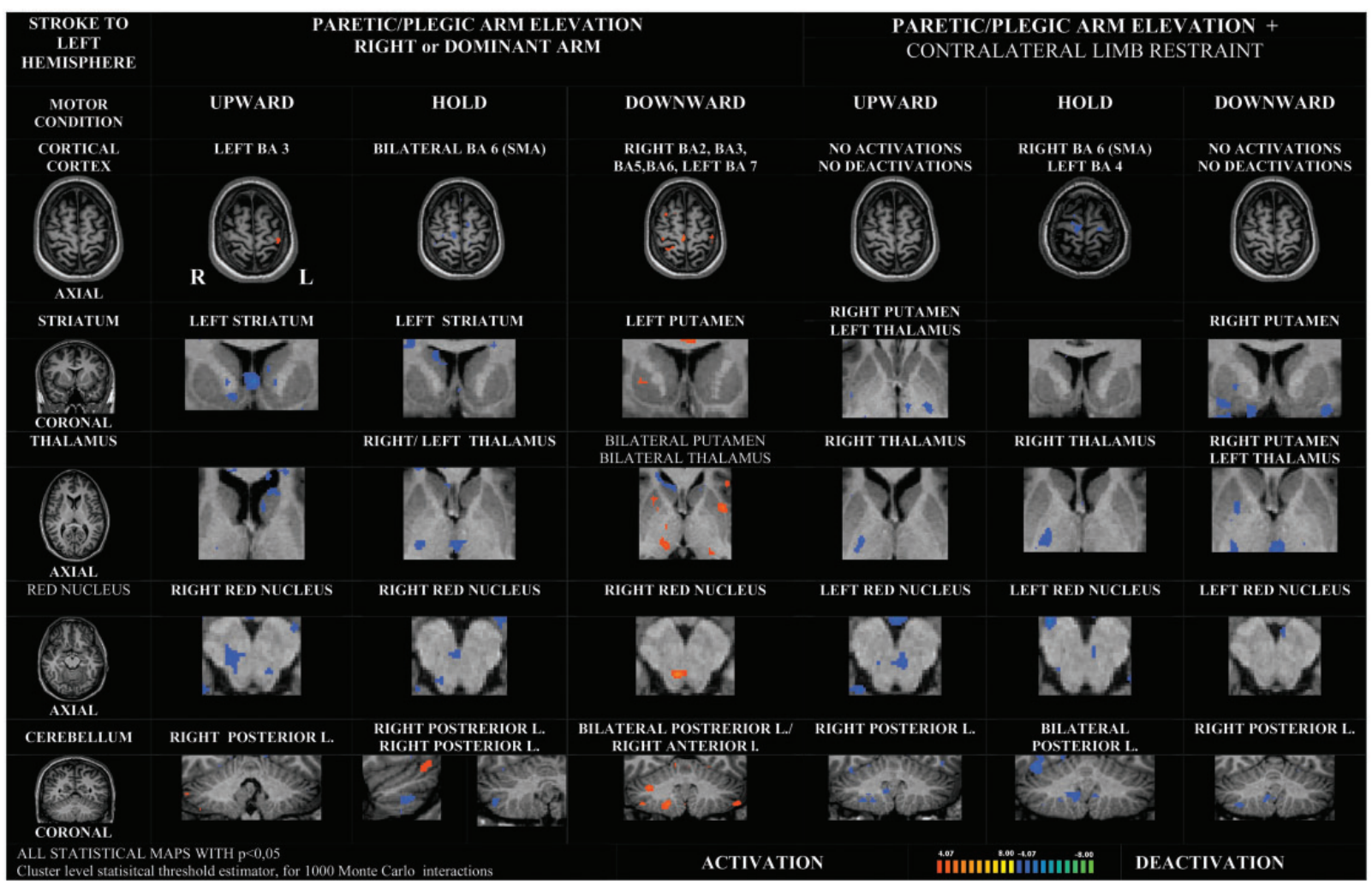

\section{Ipsilateral cerebellar activity during nonplegic/paretic $A E$}

Irrespective the side of the AE (either dominant or nondominant upper limb) ipsilateral activation of cerebellum was observed.

Supplementary Tables $6-8$ and 10 elucidate either activation or deactivation patterns (in either $\mathrm{LH}$ and $\mathrm{RH}$ stroke patients) when taking into account restraint of the paretic/plegic arm while the healthy arm is mobilized, to show the restriction-induced effects. In Supplementary tables, Appendix 1, Supplementary Table 9 lists the statistically deactivated regions in stroke patients with right cerebral hemispheric damage when only the dominant arm is elevated, without restriction.

Supplementary Table 11 provides a summary of the main results.

\section{Discussion}

The present study aimed to investigate whether motor facilitation/restraint procedures, believed to be useful in neurorehabilitation, promote neuromodulation of interhemispheric neural circuitry after a clinical episode of stroke. We focused on understanding whether the elevation of the arm when the contralateral arm is restrained leads to physiologically relevant impact in motor networks. The present study contributed to understand the underlying cortical physiology and the modulation evoked by arm restriction, as present CIMT rehabilitation approaches.

\section{The hemispheric side of the lesion influences brain activity patterns in stroke, in a dominance-dependent manner}

The different right and left hemispheric movement evoked brain activity patterns after an episode of stroke has been suggested to be dependent on the hemispheric dominance and of the side of the lesion. ${ }^{31}$ We found that contralateral upper limb restriction reduces cortical, subcortical, and cerebellar activity not only in the plegic/paretic arm during elevation but also in the "good" arm. Cerebral circuitry is accordingly modulated by both motor control-related 
Figure 4. Statistical maps of group analysis of the left hemispheric stroke patients during nondominant AE in presence/absence restriction of contralateral upper limb.

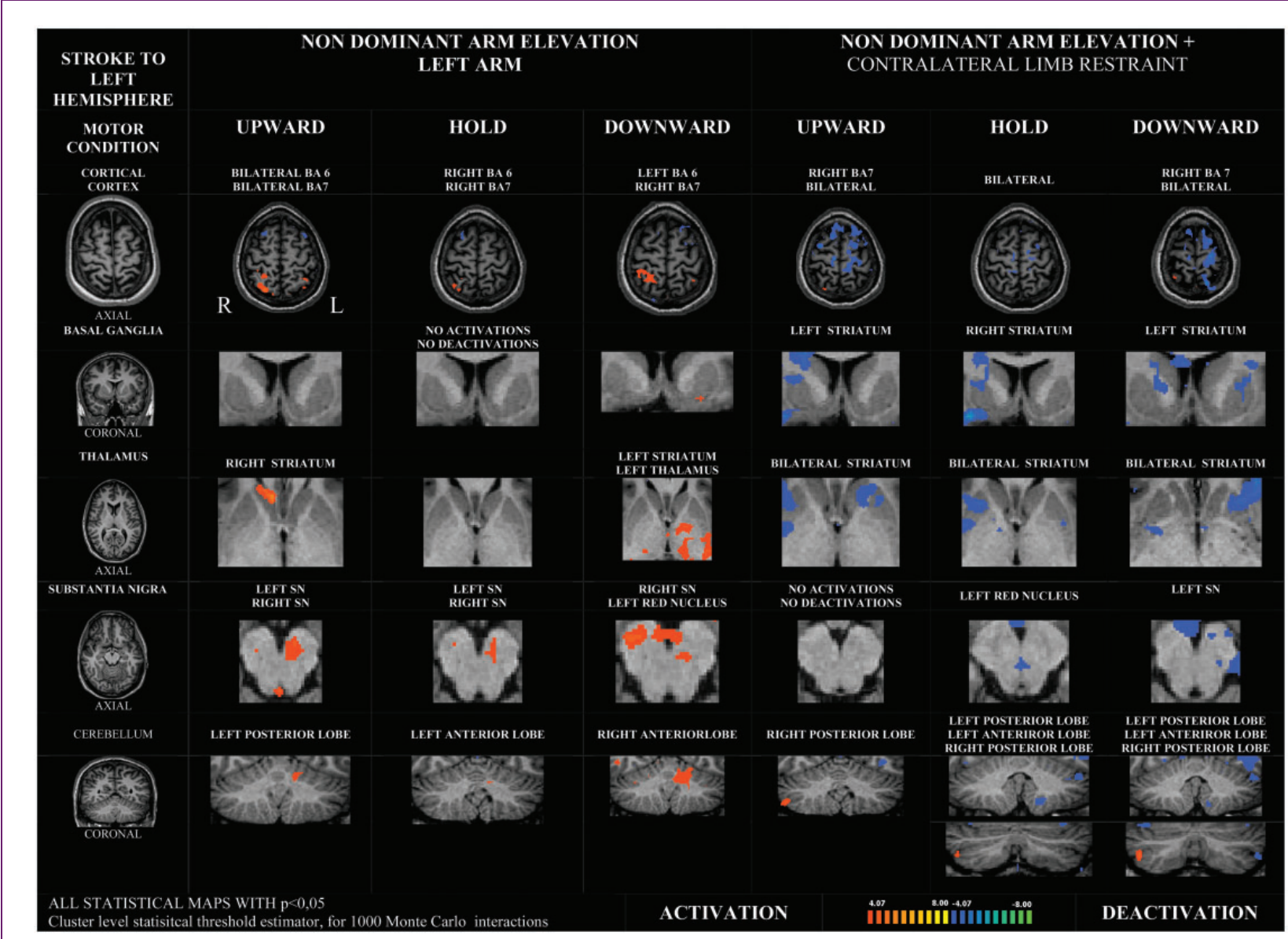

facilitation and restraining procedures, in a dominance-dependent manner. Importantly, neurophysiological responses depended strikingly on the phase of arm movement and type of motor action. These findings uncover possible brain mechanisms underlying the activation/deactivation balance which seems to be critical to drive motor recovery.

We also found bilateral cortical activation during plegic/paretic AE. However, this pattern was only observed for the right hemispheric stroke. The more localized ipsilesional/contralateral activation observed in the left hemispheric stroke patients suggests a hitherto unrecognized pattern of hemispheric dependence. Its functional significance and relation with outcome needs to be clarified in future studies.

The fact that each phase of movement recruits distinct muscle activity patterns (concentric, isometric, and eccentric) may help explain why different motor programs may have different underlying cortical and subcortical mechanisms and interhemispheric regulation.

\section{Clinical implications: neurophysiological biomarkers}

Our findings provide an important addition to understand the relationship between motor control and brain activity patterns in relation to neurorehabilitation, and in particular CIMT. The bilateral cortical activations in right hemispheric stroke lesions are interesting because such a pattern has been suggested to be predictive of more difficult motor recovery. ${ }^{11}$ Epidemiologic studies have so far not reported differences between motor recovery for left and right stroke. A previous study in healthy participants with the same motor task ${ }^{26}$ found a similar type of physiological neuromodulation in healthy controls as observed here in stroke patients, suggesting that short-term plastic mechanisms are still available in early stages. Although recent metanalyses $^{11,13}$ suggest that bilateral cortical activation was associated with jeopardized recovery and ipsilesional cortical recruitment was linked with better outcomes, this is not necessarily inconsistent with the idea that 
Figure 5. Statistical maps of group analysis of the right hemispheric stroke patients during dominant AE in presence/absence restriction of contralateral upper limb.

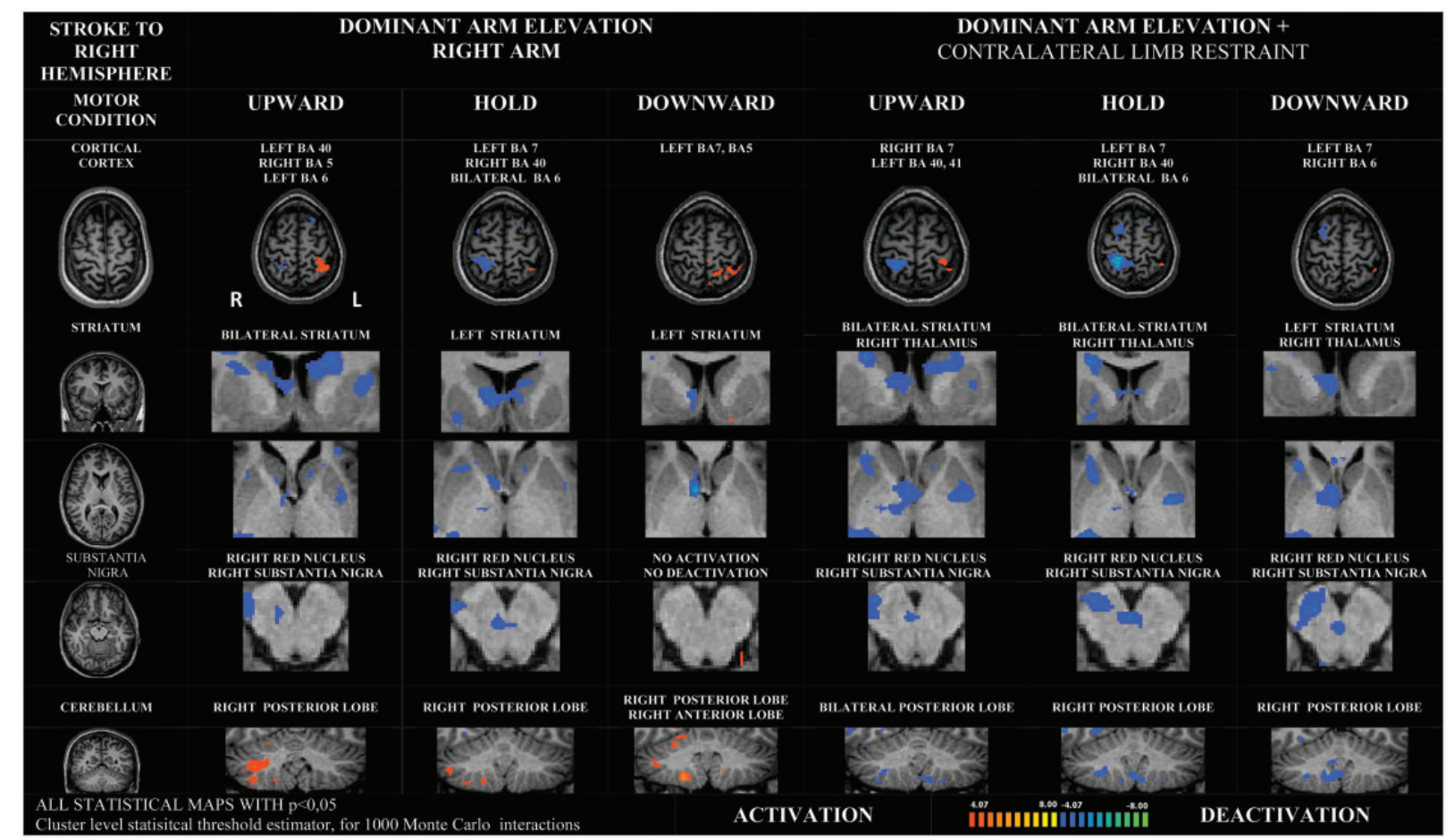

bilateral activation may precede more lateralized patterns, in a favorable manner (e.g. Nowak et al. ${ }^{32}$ ).

Our experimental results concerning the elevation of the nonaffected arm were similar and consistent with the notion of an appropriate balance between active and stop/inhibit commands of muscle activity. This idea is explained by the postulate of an ideal balance of inhibition/excitation ratio. ${ }^{9,10,33-36}$

Based on our findings, it might be useful to use transcranial magnetic stimulation (TMS) protocols ${ }^{37}$ to drive motor recovery. These clinical protocols could be based on two principles: first inhibition of overactivation and second the reestablishment of healthy brain activity patterns in accordance with the previously dominant side, before the lesion. We therefore suggest that future studies should implement protocols that value the side of stroke lesion and the hemispheric dominance. For the right hemispheric stroke we suggest that TMS should be designed to inhibit the nonaffected cerebral hemisphere aiming to inhibit bilateral cortical activation or ipsilateral overactivation. For the left hemispheric stroke we also suggest the use of an inhibiting TMS protocol to inhibit the nonaffected hemisphere to "reproduce" the cortical deactivation patterns observed in healthy participants reported in our previous study and in "good"
AE (to the right) by left stroke patients. Our work suggests the need to clinically stratify as a function of the site of lesion and future studies should further address the role of hemispheric dominance and other sources of heterogeneity.

Our results also highlight the ability to manipulate a motor condition by using movement restriction techniques that lead to changes in brain activity patterns in stroke patients.

Other important aspect to take into account in future studies is the quantification of hemispheric dominance and handedness with measures that are based on neurophysiological (brain and muscle skeletal) signals. Our results seem to confirm the theories that propose the therapeutic implementation of inhibitory modulation of the nonaffected hemisphere ${ }^{38,39} /$ less affected hemibody. ${ }^{10,40-43}$

\section{Physical therapy implications}

Our work suggests that inhibitory modulation may be a very important tool in physiotherapy, namely in CIMT, and that future interventional approaches should explore the hemisphere-dependent balance between excitation and inhibition of motor networks. 


\section{Conclusions}

We found different brain activity patterns in stroke patients who are dependent on the hemispheric side of the lesion. These findings support the asymmetry theory applied to motor control. The main implications of these findings emphasize the physiological impact of restriction, as applied in the classical CIMT therapy, in the engagement of inhibitory interactions. It sheds light on the relative role of inhibition as a counterpart of facilitation, which are important concepts in the field of motor recovery. Finally, the identified hemispheredependent functional imaging signatures can potentially be used in future diagnostic and therapeutic strategies taking into account the functional heterogeneity of neurophysiological signals that drive motor recovery after neurologic damage.

\section{Authors' contributions}

Wrote the paper: ACV, MC-B, PB, AGP, JS-F. Methods: ACV, PB, MC-B, AGP. Data selection: ACV, AGP, MC-B, GC, JS-F, AG. Data extraction: ACV. Analysis and interpretation of data: ACV, MC-B, GC, JS-F, AG, AGP.

\section{Acknowledgments}

We thank all volunteers that participated in sessions of fMRI. We also thank Carlos Ferreira, João Marques, and Bruno Quendera for their help with fMRI scanning. We thank occupational therapists of Garcia de Orta Hospital, Almada, Portugal for their help in construction of Cellacast ${ }^{\circledR}$ splint for restriction flexion on elbow joint. We also thank Miguel Patrício for statistical advice.

\section{Declaration of conflicting interests}

The author(s) declared no potential conflicts of interest with respect to the research, authorship, and/or publication of this article.

\section{Funding}

The author(s) disclosed receipt of the following financial support for the research, authorship, and/or publication of this article: Individual grant of Foundation of Science and Technology: SFRH/BD/63848/2009 to Ana C Vidal, and CENTRO-07-ST24-FEDER-00205, FCT UID/NEU/04539/ 2013.

\section{ORCID iD}

João Sargento-Freitas (D) http://orcid.org/0000-0003-46655697

\section{References}

1. Duncan PW, Zorowitz R, Bates B, et al. Management of adult stroke rehabilitation care: a clinical practice guideline. Stroke 2005; 36: e100-e143.
2. Kwakkel G, Kollen BJ, van der Grond $\mathbf{J}$ and Prevo AJ. Probability of regaining dexterity in the flaccid upper limb: impact of severity of paresis and time since onset in acute stroke. Stroke 2003; 34: 2181-2186.

3. Feigin VL, Forouzanfar MH, Krishnamurthi R, et al. Global and regional burden of stroke during 19902010: findings from the Global Burden of Disease Study 2010. Lancet 2014; 383: 245-254.

4. Go AS, Mozaffarian D, Roger VL, et al. Executive summary: heart disease and stroke statistics - 2014 update: a report from the American Heart Association. Circulation 2014; 129: 399-410.

5. Lackland DT, Roccella EJ, Deutsch AF, et al. Factors influencing the decline in stroke mortality: a statement from the American Heart Association/American Stroke Association. Stroke 2014; 45: 315-353.

6. Gomes A, Rinehart N, Greenham M and Anderson V. A critical review of psychosocial outcomes following childhood stroke (1995-2012). Dev Neuropsychol 2014; 39: 9-24.

7. Craig LE, Wu O, Bernhardt $\mathrm{J}$ and Langhorne $\mathrm{P}$. Approaches to economic evaluations of stroke rehabilitation. Int J Stroke 2014; 9: 88-100.

8. Ma VY, Chan L and Carruthers KJ. The incidence, prevalence, costs and impact on disability of common conditions requiring rehabilitation in the US: stroke, spinal cord injury, traumatic brain injury, multiple sclerosis, osteoarthritis, rheumatoid arthritis, limb loss, and back pain. Arch Phys Med Rehabil 2014; 95: 986-995.e1.

9. Pascual-Leone A, Amedi A, Fregni F and Merabet LB. The plastic human brain cortex. Аппи Rev Neurosci 2005; 28: $377-401$.

10. Takeuchi $\mathrm{N}$ and Izumi $\mathrm{S}$. Maladaptive plasticity for motor recovery after stroke: mechanisms and approaches. Neural Plast 2012; 2012: 359728.

11. Favre I, Zeffiro TA, Detante O, Krainik A, Hommel M and Jaillard A. Upper limb recovery after stroke is associated with ipsilesional primary motor cortical activity: a meta-analysis. Stroke 2014; 45: 1077-1083.

12. Rehme AK and Grefkes C. Cerebral network disorders after stroke: evidence from imaging-based connectivity analyses of active and resting brain states in humans. J Physiol 2013; 591: 17-31.

13. Grefkes $\mathrm{C}$ and Fink GR. Connectivity-based approaches in stroke and recovery of function. Lancet Neurol 2014; 13: 206-216.

14. Michaelsen SM, Dannenbaum R and Levin MF. Taskspecific training with trunk restraint on arm recovery in stroke: randomized control trial. Stroke 2006; 37: 186-192.

15. Langhorne $\mathrm{P}$, Bernhardt $\mathrm{J}$ and Kwakkel G. Stroke rehabilitation. Lancet 2011; 377: 1693-1702.

16. Veerbeek JM, van Wegen E, van Peppen R, et al. What is the evidence for physical therapy poststroke? A systematic review and meta-analysis. PLoS One 2014; 9: e87987.

17. Murayama T, Numata K, Kawakami T, et al. Changes in the brain activation balance in motor-related areas after constraint-induced movement therapy; a longitudinal fMRI study. Brain Inj 2011; 25: 1047-1057. 
18. Wittenberg GF and Schaechter JD. The neural basis of constraint-induced movement therapy. Curr Opin Neurol 2009; 22: 582-588.

19. Hindle KB, Whitcomb TJ, Briggs WO and Hong J, Proprioceptive neuromuscular facilitation (PNF): its mechanisms and effects on range of motion and muscular function. J Hum Kinet 2012; 31: 105-113.

20. Levin MF and Panturin E. Sensorimotor integration for functional recovery and the Bobath approach. Motor Control 2011; 15: 285-301.

21. Stefanovic B, Warnking JM and Pike GB. Hemodynamic and metabolic responses to neuronal inhibition. Neuroimage 2004; 22: 771-778.

22. Nirkko AC, Ozdoba C, Redmond SM, et al. Different ipsilateral representations for distal and proximal movements in the sensorimotor cortex: activation and deactivation patterns. Neuroimage 2001; 13: 825-835.

23. Hayes DJ and Huxtable AG. Interpreting deactivations in neuroimaging. Front Psychol 2012; 3: 27.

24. Allison JD, Meador KJ, Loring DW, Figueroa RE and Wright JC. Functional MRI cerebral activation and deactivation during finger movement. Neurology 2000; 54: $135-142$.

25. Grefkes C, Eickhoff SB, Nowak DA, Dafotakis M and Fink GR. Dynamic intra- and interhemispheric interactions during unilateral and bilateral hand movements assessed with fMRI and DCM. Neuroimage 2008; 41: 1382-1394.

26. Vidal AC, Banca P, Pascoal AG, Cordeiro G, SargentoFreitas J and Castelo-Branco M. Modulation of cortical interhemispheric interactions by motor facilitation or restraint. Neural Plast 2014; 1-8. doi: 10.1155/2014/210396.

27. Oldfield RC. The assessment and analysis of handedness: the Edinburgh inventory. Neuropsychologia 1971; 9: 97-113.

28. Sims JR, Gharai LR, Schaefer PW, et al. ABC/2 for rapid clinical estimate of infarct, perfusion, and mismatch volumes. Neurology 2009; 72: 2104-2110.

29. Kunst MM and Schaefer PW. Ischemic stroke. Radiol Clin North Am 2011; 49: 1-26.

30. Brooks DJ. The role of the basal ganglia in motor control: contributions from PET. J Neurol Sci 1995; 128: $1-13$.
31. Mani S, Mutha PK, Przybyla A, Haaland KY, Good DC and Sainburg RL. Contralesional motor deficits after unilateral stroke reflect hemisphere-specific control mechanisms. Brain 2013; 136: 1288-1303.

32. Nowak DA, Grefkes C, Ameli $M$ and Fink GR. Interhemispheric competition after stroke: brain stimulation to enhance recovery of function of the affected hand. Neurorehabil Neural Repair 2009; 23: 641-656.

33. Nudo RJ. Plasticity. NeuroRx 2006; 3: 420-427.

34. Nudo RJ, Plautz EJ and Frost SB. Role of adaptive plasticity in recovery of function after damage to motor cortex. Muscle Nerve 2001; 24: 1000-1019.

35. Allred RP, Cappellini CH and Jones TA. The "good" limb makes the "bad" limb worse: experience-dependent interhemispheric disruption of functional outcome after cortical infarcts in rats. Behav Neurosci 2010; 124: 124-132.

36. Takeuchi N, Oouchida Y and Izumi S. Motor control and neural plasticity through interhemispheric interactions. Neural Plast 2012; 2012: 823285.

37. Fregni F, Boggio PS, Mansur CG, et al. Transcranial direct current stimulation of the unaffected hemisphere in stroke patients. Neuroreport 2005; 16: 1551-1555.

38. Riecker A, Groschel K, Ackermann H, Schnaudigel S, Kassubek $\mathbf{J}$ and Kastrup A. The role of the unaffected hemisphere in motor recovery after stroke. Hum Brain Mapp 2010; 31: 1017-1029.

39. Rehme AK, Fink GR, von Cramon DY and Grefkes C. The role of the contralesional motor cortex for motor recovery in the early days after stroke assessed with longitudinal FMRI. Cereb Cortex 2011; 21: 756-768.

40. Cirstea MC and Levin MF. Compensatory strategies for reaching in stroke. Brain 2000; 123: 940-953.

41. Takeuchi $\mathrm{N}$ and Izumi S. Rehabilitation with poststroke motor recovery: a review with a focus on neural plasticity. Stroke Res Treat 2013; 2013: 128641.

42. Kerr AL, Wolke ML, Bell JA and Jones TA. Post-stroke protection from maladaptive effects of learning with the non-paretic forelimb by bimanual home cage experience in C57BL/6 mice. Behav Brain Res 2013; 252: 180-187.

43. Kapur N. Paradoxical functional facilitation in brainbehaviour research. A critical review. Brain 1996; 119: 1775-1790. 